\title{
On the Structure of Au/Pd Bimetallic Nanoparticles
}

\author{
Sergio J. Mejía-Rosales,* Carlos Fernández-Navarro, Eduardo Pérez-Tijerina, \\ Douglas A. Blom, Lawrence F. Allard, and Miguel José-Yacamán ${ }^{\dagger}$
}

Facultad de Ciencias Físico-Matemáticas, Universidad Autónoma de Nuevo León, San Nicolás de los Garza, Nuevo León, México 66450, Materials Science and Technology Division, Oak Ridge National Laboratory, Oak Ridge, Tennessee 37831, and Chemical Engineering Department and Texas Advanced Materials Center, The University of Texas at Austin, Austin, Texas 78712

Received: September 26, 2006; In Final Form: November 8, 2006

\begin{abstract}
We performed a study on bimetallic Au/Pd nanoparticles using aberration corrected electron microscopy along with molecular dynamics simulations to investigate the features of specific atomic sites at the surface, which can be related to the high catalytic activity properties of the particles. The calculations mimic the growth of nanoparticles through a cooling process from a molten solid to a crystalline structure at room temperature. We found that the final structure for the alloy particles is neither a cuboctahedral nor an icosahedral, but a complex structure that has a very rough surface and unique isolated Pd sites surrounded by Au atoms. We also found that there is predominance of three specific Pd sites at the surface, which can be directly related to the catalytic activity of the nanoparticles.
\end{abstract}

\section{Introduction}

It is considered that nanotechnology applied to catalysis will produce a new generation of catalysts by design, an approach needed to provide a more precise control of selectivity and activity in catalytic reactions. Of especially strong current interest for such studies is gold metal, whose catalytic properties have recently shown high promise.

After the key findings of Hutchings on the properties of $\mathrm{Au}$ as catalyst for ethyne hydrochlorination ${ }^{1}$ and Haruta on $\mathrm{Au}$ as catalyst for $\mathrm{CO}$ oxidation at low temperature ${ }^{2}$ there has been a dramatic increase in research on gold catalysts. It is likely that it will be possible in the near future to tailor gold catalysts so they are optimized for reactions such as hydrogen production, fuel cells, fine chemicals, sensors, and the like.

The first well-established application of gold is the use of bimetallic $\mathrm{Au}-\mathrm{Pd}$ catalysts for the production of vinyl acetate monomers (VAM) from ethane, acetic acid, and oxygen. Enache et al. ${ }^{3}$ have recently shown that $\mathrm{Au}-\mathrm{Pd}$ catalysts supported on $\mathrm{TiO}_{2}$ give very high turnover frequencies for the oxidation of alcohols. In a recent paper, Chen et al. have reported that the high activity for VA formation in the $\mathrm{Au}-\mathrm{Pd}$ system might be explained by the presence on the surface of $\mathrm{Pd}$ atoms that are isolated by the gold atoms. ${ }^{4}$ This is based on the fact that the rate of VA formation is enhanced in the $\mathrm{Au}-\mathrm{Pd}$ (100) plane with respect to the $\mathrm{Au}-\mathrm{Pd}$ (111) plane, suggesting that the critical site is a pair of $\mathrm{Pd}$ atoms spaced at $\sim 3.3 \AA$. The main role of the gold is suggested to be the stabilization of those sites on the surface.

In the present work we use high-resolution bright-field transmission electron microscopy (TEM) and aberration-corrected high-angle annular dark-field scanning transmission electron microscopy (HAADF-STEM, with resolution $<1 \AA$ ), coupled with molecular dynamics (MD) calculations, to study $\mathrm{Au}-\mathrm{Pd}$ nanoparticles. Our study contributes to give a deeper

* Address correspondence to this author. E-mail: smejia@ @fcfm.uanl.mx.

E-mail: yacaman@che.utexas.edu. insight of the understanding of the nature of the catalytic sites, and to suggest a way to improve both the selectivity and activity of gold-based catalysts.

\section{Experimental Methods and Calculations}

The basic polyol method ${ }^{5}$ was followed to obtain bimetallic nanoparticles passivated with poly(vinylpyrrolidone) (PVP). Hydrogen tetrachloroaurate $\left(\mathrm{HAuCl}_{4}\right)(\mathrm{III})$ hydrate $(99.99 \%)$ and palladium chloride $\left(\mathrm{PdCl}_{2}\right)(99.99 \%)$ were purchased from Sigma Aldrich; all the materials were used without any further treatment. A $0.4-\mathrm{g}$ sample of PVP was dissolved in $50 \mathrm{~mL}$ of EG under vigorous stirring, and reflux heated until the desired temperature was reached (working temperatures ranged from 100 to $190{ }^{\circ} \mathrm{C}$ ). When preparing the bimetallic nanoparticles, the following criteria were used: after complete dissolution of PVP in EG, $2 \mathrm{~mL}$ of an aqueous solution $(0.05 \mathrm{M})$ of $\mathrm{PdCl}_{2}$ was added. One minute after the change of color of the solution from yellow to dark brown was observed, $1 \mathrm{~mL}$ of an aqueous solution of $\mathrm{HAuCI}_{4}(0.1 \mathrm{M})$ was added to the system. The reaction was carried out for $3 \mathrm{~h}$ at constant temperature. This order and time between precursor additions was selected after trying several other possibilities. The one chosen for this work produced the smallest nanoparticle average size and the smallest standard deviation for a synthesis performed at $140{ }^{\circ} \mathrm{C}$. With this procedure we prepared a range of alloys with atomic compositions $\mathrm{AuPd}, \mathrm{Au}_{3} \mathrm{Pd}$, and $\mathrm{AuPd}_{3}$.

A drop of the solution was deposited on a carbon holey film for TEM observation. High-resolution TEM images were acquired with a JEOL 2010F field-emission TEM, a FEI Tecnai 20 Super-twin field-emission TEM, and a Jeol 4000 EX. The images were obtained at the optimum (Scherzer) defocus setting in all of the TEM instruments. HA-ADF STEM images were acquired by using a JEOL 2200FS instrument equipped with an aberration corrector on the incident probe (CEOS $\mathrm{GmbH}$, Heidelberg, Germany), which provides a nominal resolution of below $1 \AA$ at an acceptance semiangle of $26.5 \mathrm{mrad}$.

We performed a set of molecular dynamics simulations on the canonical (NVT) ensemble, with a Nosé-Hoover thermostat 


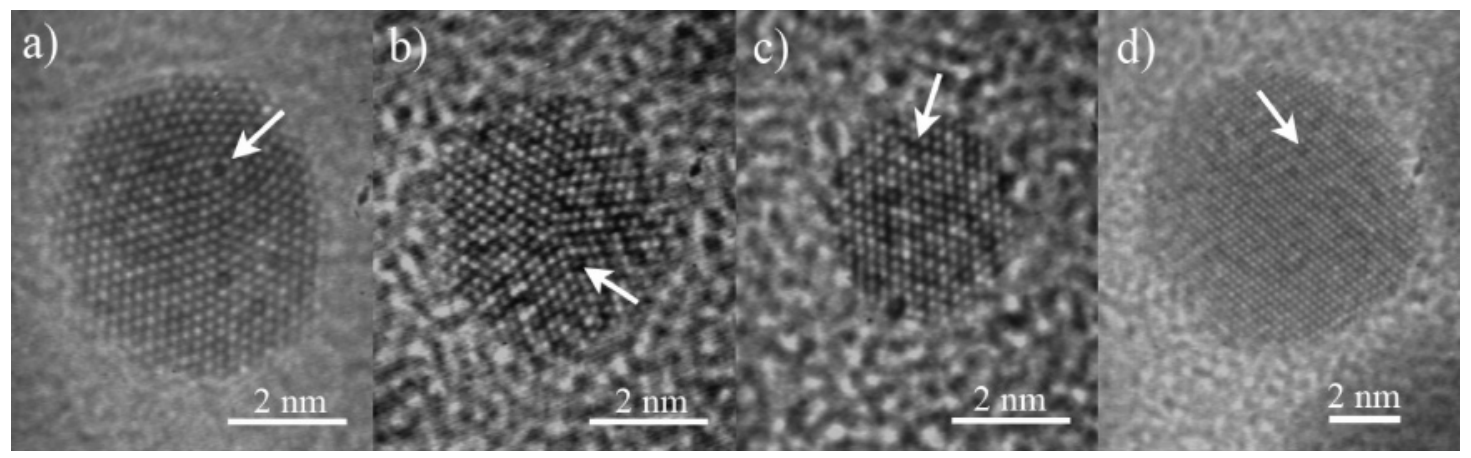

Figure 1. Bright-field TEM images of AuPd nanoparticles prepared by the basic polyol method as described in the text. The arrows show surface sites with vacancies.

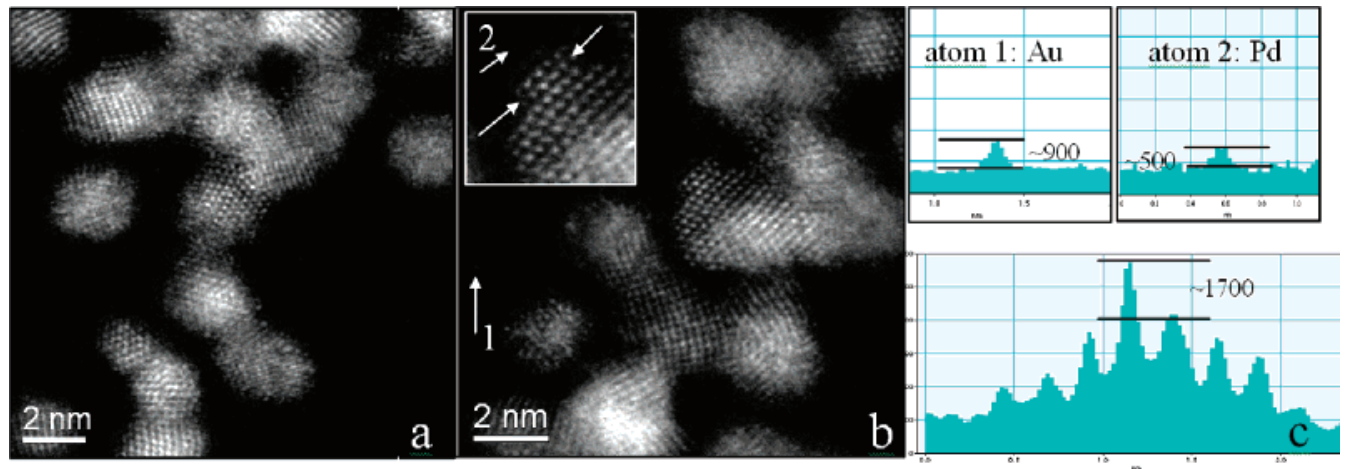

Figure 2. HA-ADF images of typical $\mathrm{AuPd}_{3}$ nanoparticles (a and b). Single atom traces (c, top) suggest Pd intensity of 500 counts vs Au intensity of 900 counts. The profile of the atom row indicated on the inset (c, bottom) shows a central column roughly triple the intensity of the adjacent column, presuming all $\mathrm{Pd}$ atoms. This suggests the presence of one or more Au atoms in the central column.

that controls the temperature of the thermal bath through the addition of an extra degree of freedom on the Hamiltonian; the dynamics of this additional coordinate rescales the atomic velocities, forcing the system to keep the temperature constant. ${ }^{6}$ The equations of motion were integrated by using the velocity Verlet algorithm, storing the velocities and forces acting on the atoms at every $\Delta t .^{7} \mathrm{We}$ used the MD code DL_POLY $v 2.14$ to perform the MD calculations. ${ }^{8}$ To determine the appropriate value of $\Delta t$, we undertook a series of runs at different time steps, monitoring the stability of the Hamiltonian, and found that the best compromise between Hamiltonian conservation and optimization of computer time was obtained using a value of 1.4 fs. Every system was thermalized by running a simulation 150 ps long to relax the structures. In total, each simulation run was $4.9 \mathrm{~ns}$ long. The interatomic potential used in our simulations is the one developed by Raffi-Tabar and Sutton, in a generalization of the Sutton and Chen potential for the case of FCC metallic alloys. ${ }^{9}$ This model has been used successfully to simulate the dynamics and structural properties of different alloys, such as $\mathrm{Au}-\mathrm{Cu},{ }^{10} \mathrm{Cu}-\mathrm{Ag}$, and $\mathrm{Cu}-\mathrm{Ni} .{ }^{11}$ We used the functional form and parameters for $\mathrm{Au}-\mathrm{Pd}$ as reported in ref 12.

\section{Results and Discussion}

3.1. Experimental Measurements. We have studied the Au/ Pd nanoparticles using TEM. Scherzer defocus TEM images for the case of AuPd are shown in Figure 1, panels a-d. In these pictures a very pronounced black and white contrast can be seen, and there are many sites that seem to indicate a surface vacancy (see, for example, the site marked with an arrow in Figure 1a). The same kind of contrast is observed for the particles shown in Figure $1 b-d$. The particle in Figure $1 b$ corresponds to a decahedron. The particles in Figure 1c,d correspond to a cuboctahedron, and show the typical FCC structure for the particle. We believe that these sites correspond to extended point defects on the surface such as kinks, edges, and group vacancies.

A second set of observations was made by using HAADFSTEM. Figure 2 shows three typical images of AuPd nanoparticles. In this case the interpretation of the atom images is more straightforward, since the image intensity $(I)$ is $I \approx Z^{1.6}(Z=$ atomic number). The roughness of the surface, due to the presence of extended point defects, can also be seen clearly. The presence of $\mathrm{Au}$ atoms in columns of $\mathrm{Pd}$ atoms can therefore be inferred by observations on the contrast of the atomic columns. Our experimental preparation method, which involves sequential reduction of the two metals, results in an alloy structure, in contrast with the case of simultaneous reduction that results in a core shell structure in agreement with previous work. ${ }^{13}$

Figure 2 shows HAADF images of nanoparticles of $\mathrm{AuPd}_{3}$. It is clear that some atomic columns are significantly brighter that adjacent columns, as is shown by the contrast of the arrowed atom row in the inset of Figure 2b. Figure 2c (top) shows intensity traces over two single atoms labeled 1 and 2 . These are typical of several such traces in Figure $2 b$, and suggest that the higher intensity atom is $\mathrm{Au}$, and the lower one is Pd. Since the intensity is proportional to $Z^{1.6}$, the relative single atom intensities should be $791.6 / 461.6=1087 / 458=2.37$. It is very reasonable to assume that the more intense atoms correspond to gold and the less intense atoms correspond to $\mathrm{Pd}$, since the intensity ratio is 1.8 , a reasonable approximation of the expected ratio of 2.37, given the low sampling and low signal above background. With regard to the bright central column then, if all $\mathrm{Pd}$, it represents an unreasonable number (at least 3 ) of $\mathrm{Pd}$ atoms extending above the general surface. This is not favored 


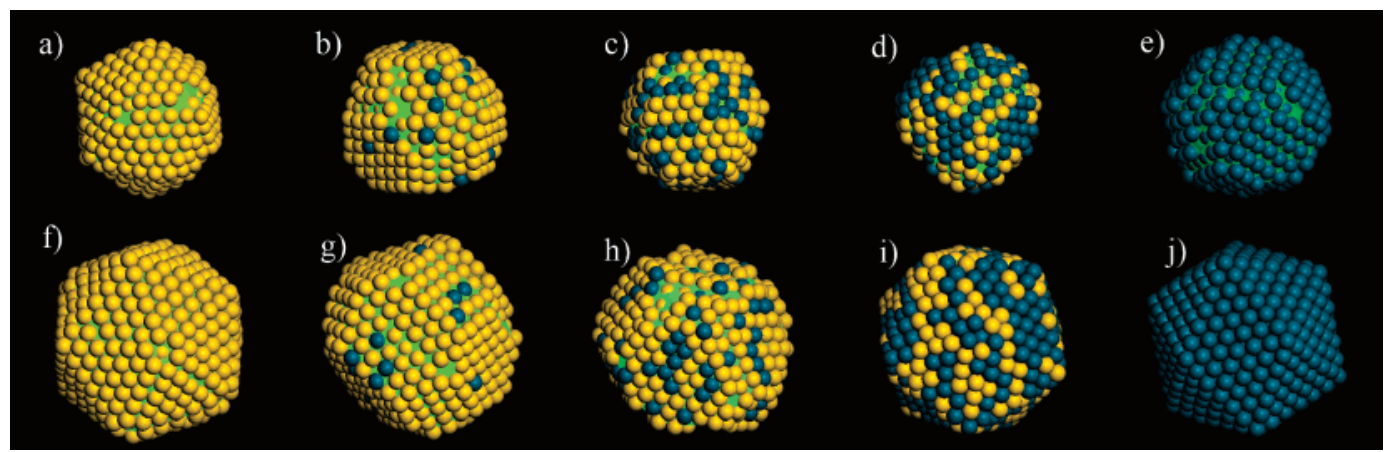

Figure 3. Final configurations at $300 \mathrm{~K}$, obtained after the cooling process. For the Pd and pure Au particles the icosahedral geometry is very well defined; the alloy particles present more complicated shapes. It can be noted the tendency of Au to occupy the surface of the particle. The atoms located at the core have been hidden to emphasize the structure at the surface. The configurations in the upper row correspond to 561-atom particles, and those of the lower row correspond to 923 -atom particles. a and f: pure gold. b and g: $\mathrm{Au}_{3} \mathrm{Pd} . \mathrm{c}$ and h: AuPd. $\mathrm{d}$ and i: $\mathrm{AuPd}_{3} . \mathrm{e}$ and j: pure $\mathrm{Pd}$.

energetically; it is likely therefore that one or more $\mathrm{Au}$ atoms contribute to the intensity of this column. HAADF image computations of model particles correlating to experimental images will be the subject of a followup paper.

3.2. Molecular Dynamics Calculations. To explain the experimental results, we performed a series of MD simulations for different $\mathrm{Au}-\mathrm{Pd}$ nanoparticles. We ran simulations on particles of two different sizes, 561 and 923 atoms. The identity of the atoms in the alloys was randomly generated for all cluster concentrations, such that the two atomic species were uniformly distributed along the particles. Five different concentrations were used: pure $\mathrm{Au}, \mathrm{Au}_{3} \mathrm{Pd}, \mathrm{AuPd}, \mathrm{AuPd}_{3}$, and pure Pd. The final liquid configuration from a previously computed heating series ${ }^{14}$ was used as the initial configuration for the freezing series. This choice of the initial configuration is arbitrary but not improper, in the sense that any configuration obtained at thermal equilibrium would be equally useful, since at high temperatures the atoms are not bound to equilibrium lattice sites, the shape of the particle is constantly changing, and any typical configuration, including the last configuration in the previous run, can be used without producing computation artifacts; nevertheless, it is known that different initial configurations from a molten state may lead to significant differences both on the freezing temperature and on the final configuration of the run (see, for example, ref 15). For each nanoparticle we obtained two series of simulations, each series formed by runs performed within a temperature range from 980 to $300 \mathrm{~K}$, in intervals of $20 \mathrm{~K}$. The starting configuration at a given $T$ was taken from the final state of the run made at the immediate upper temperature, with the appropriate scaling of the atomic velocities to match the corresponding value of $T$. The resulting final structures at 300 $\mathrm{K}$ are shown in Figure 3 for the different relative concentrations of $\mathrm{Au}-\mathrm{Pd}$, where we note that the resolidified structures in the pure monometallic particles present well-defined geometries. We can also note from the images of the alloy particles after freezing the tendency of gold atoms to populate the surface.

The resulting caloric curves are drawn in Figure 4, where the freezing transition can be located for each case. As expected, the freezing temperatures, corresponding to the values of $T$ where the curves show a pronounced discontinuity, depend on the relative concentrations of the atomic species.

To investigate the details of the atomic arrangements, we calculated the value of the atomic order parameter $q_{6}$, defined by

$$
q_{6}(i)=\left(\frac{4 \pi}{13} \sum_{m=-6}^{6}\left|q_{6 m}(i)\right|^{2}\right)^{1 / 2}
$$

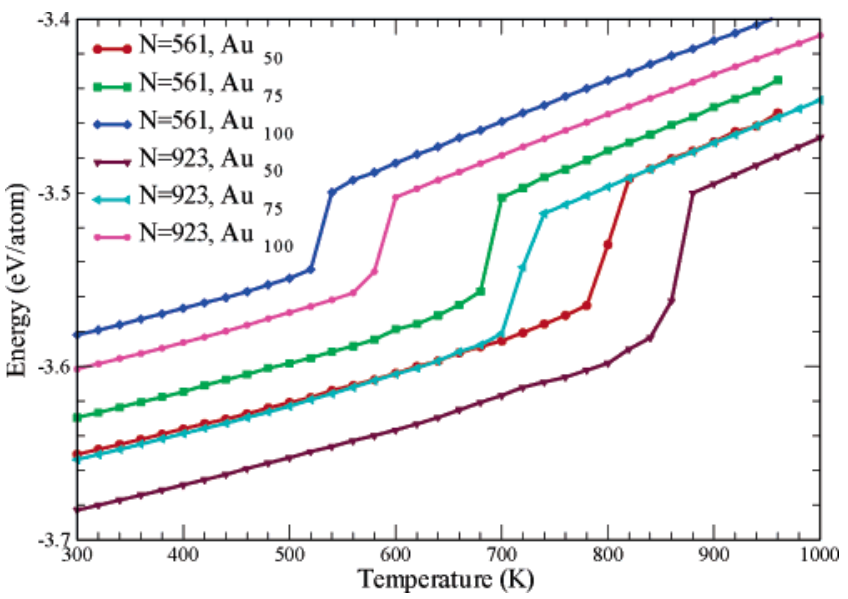

Figure 4. Caloric curves obtained by molecular dynamics for the different simulated particles. The abrupt changes of slope in the curves indicate the freezing transition. The points at $300 \mathrm{~K}$ correspond to the structures shown in Figure 3.

where

$$
q_{6 m}(i)=\frac{\sum_{j=1}^{N_{n}(i)} Y_{6 m}\left(\mathbf{r}_{i j}\right)}{N_{n}(i)}
$$

are the average of the spherical harmonics of the bonds of the atom with the neighbors. This definition is an appropriate measure of the coordination for each atom. ${ }^{16}$ On the basis of the values of $q_{6}$, the atoms in the particles were identified according to their local structure; thus, it was possible to differentiate the atoms with a FCC structure from those with an HCP or a 5-fold structure. This is shown in Figure 5. In the $\mathrm{Au}$ and AuPd particles, the structure is formed by parallel alternating groups of rows of HCP (red spheres in the figure) and FCC atoms (gray spheres), whereas in the $\mathrm{Au}_{3} \mathrm{Pd}$ system, the particle is structured into several FCC regions separated by HCP-like frontiers. For all of the 561-atom particles simulated in our study, the constructions formed after freezing differ from previous reported results of freezing of larger pure gold nanoparticles, where most of the frozen clusters attained an icosahedral structure. For the 923-atom pure gold nanoparticle, in contrast to the 561-atom particle, the resulting structure after freezing was indeed icosahedral. The runs for a 923-atom pure palladium particle gave also an icosahedron as a final structure, but for the 923-atom alloys $\mathrm{Au}_{3} \mathrm{Pd}$ and $\mathrm{AuPd}$, the final structures 


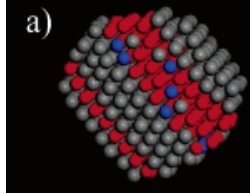

f)

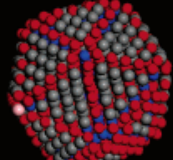

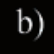

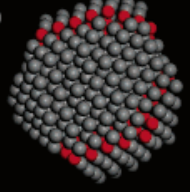

g)

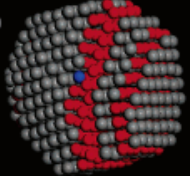

c)

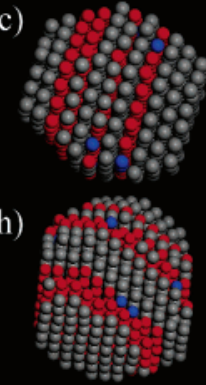

d)

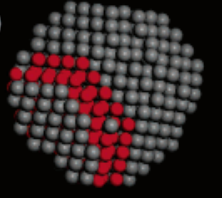

i)

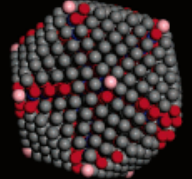

e)

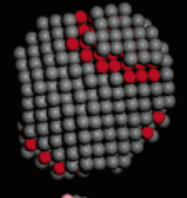

j)

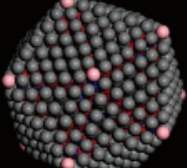

Figure 5. Final configurations at $300 \mathrm{~K}$, obtained after the cooling process. Here, the color of the atoms represents different values of the local order parameter $q_{6}$. Gray spheres represent atoms with an FCC local structure, red spheres are atoms in an HCP environment, pink atoms have a $I_{h}$ local structure, and blue atoms indicate sites with 5-fold $\left(D_{h}\right)$ symmetry. The configurations in the upper row correspond to 561-atom particles, and those of the lower row correspond to 923-atom particles. a and f: pure gold. b and g: $\mathrm{Au}_{3} \mathrm{Pd} . \mathrm{c}$ and h: $\mathrm{AuPd}$. $\mathrm{d}$ and i: $\mathrm{AuPd}$. e and j: pure Pd.

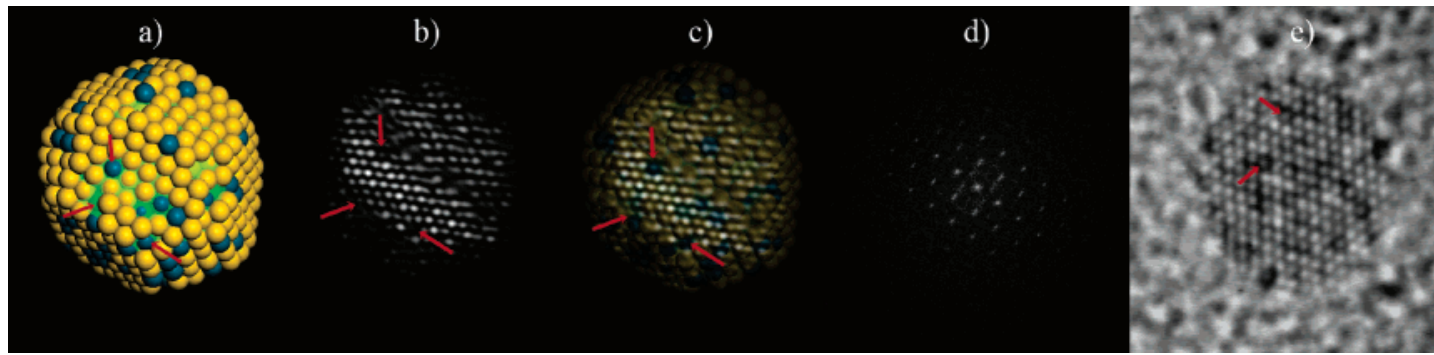

Figure 6. (a) Final configuration for the AuPd 923-atom particle, where the surface roughness can be noted; (b) the corresponding simulated TEM image; (c) overlap of parts a and b; (d) calculated FFT pattern for the simulated particle; (d) TEM image of a real particle of a size similar to that of the simulated particle that shows the same kind of features at the surface.

resembled those obtained in the 561-atom systems. This can be thought of as evidence that the final geometry of the nanoparticles and the local structure after the particle is formed may be determined at least in some part by the relative concentration of the species. Indeed, we can note from the comparison of Figures 3 and 5 that in those nanoparticles being formed by bands of FCC material alternating with bands of HCP material, the surface is rougher than in the particles without alternating bands. A similar appearance of FCC planes embedded on HCP structures and of HCP planes in FCC structures was previously reported and discussed by Chushak and Bartell. ${ }^{17}$ The final structure of the bimetallic particle shows in the last two layers a large number of defects; for instance, we can identify vacancies that produce strain around the neighboring atoms. We also identify kinks and edges. We believe that those defects are the result of the faults on the stacking on the atoms in the inner layers of the particle.

3.3. MD and STEM Comparisons. We calculated the corresponding HR-TEM images of the final structures, and we present some of the results in Figure 6. We chose in particular the case of the 923-atom AuPd particle since, as can be seen in Figure 3, this is one of the simulated particles with a less defined geometry, and with vacancies and excess $\mathrm{Au}$ at the surface. We use the atomic positions of the final structure obtained with the MD calculations to feed into the multislice program SimulaTEM, to simulate electron microscopy images for different orientations of the sample with respect to the electron beam. For the orientation shown in Figure 6a, the resulting simulated image and its corresponding FFT (showing image periodicities) are shown in Figure 6, panels $b$ and $d$, respectively. The simulated TEM image was taken in the Scherzer defocus $(-405.45 \AA)$, at a voltage of $400 \mathrm{k} \mathrm{eV}$, with a spherical aberration of $1 \mathrm{~mm}$, and a defocus spread of $38 \AA$. The variation of the image contrast was controlled by a small tilting angle $\theta$, that for the image shown in the figure was set at $\theta=0.72^{\circ}$. Under these conditions, contrast variations producing black and

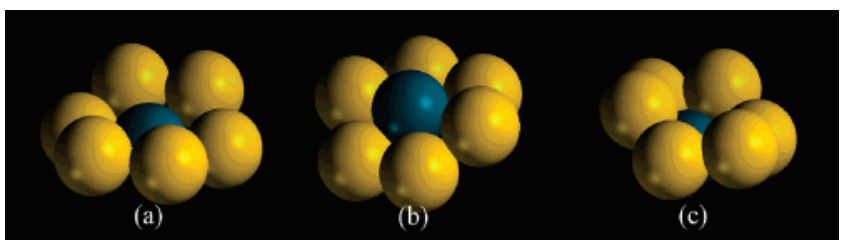

Figure 7. Typical surface sites formed by a Pd atom surrounded by a hexagonal array of Au atoms. The Pd atom is (a) roughly at the same height as the Au atoms, (b) at the top of the Au hexagonal array, and (c) one layer below the Au hexagonal array.

white atom columns are clearly observed in the images; these variations can be thought of as due to thickness variations on the atomic columns and correspond to surface roughness with vacancies and kink sites as the ones shown in the models, as can be noted in Figure 6c, built by the overlap of panels a and $b$ of Figure 6 . Figure 6 e reproduces one of the real particles presented in Figure 1, to compare the surface features with those observed in the simulated TEM images.

The STEM HAADF images clearly show the special roughness sites. In particular, we have observed a site that is the most common one; this kind of site is pictured in Figure 7. These sites can be described as an hexagonal array of gold atoms with a Pd atom in the center. However, three positions of the Pd are possible, which are (i) the atom one layer below, (ii) the atom in the same layer, and (iii) the Pd at the top of an hexagonal array. It should be noted that in the hexagonal array, the gold atoms surrounding the $\mathrm{Pd}$ atom are not on the same plane. Doing a statistical analysis of the surface we found that the sites shown in Figure 7 are the most frequently observed in the simulated structure. These sites are also observed in many experimental images, just as those shown in Figure 1. It should be noted that it is also possible to have the same kind of sites but with the atomic species inverted, i.e., positions of $\mathrm{Au}$ and $\mathrm{Pd}$ in Figure 7 can be interchanged. We notice that in the alloy, the distance 
between individual Pd atoms becomes $\sim 5.6 \AA$, which is larger than that obtained by Chen et al. ${ }^{4}$

\section{Conclusions}

We have performed a systematic study of the structure of bimetallic nanoparticles of $\mathrm{Au} / \mathrm{Pd}$ using theoretical calculations coupled with experimental data. Alloy nanoparticles with atomic compositions $\mathrm{AuPd}, \mathrm{Au}_{3} \mathrm{Pd}$, and $\mathrm{AuPd}_{3}$ were synthesized by sequential reduction with the polyol method, and analyzed by TEM and HAADF-STEM. The cooling calculation presented in this work mimics the way in which nanoparticles are grown in many experimental conditions. When we performed a molecular dynamics cooling process of a particle containing only one kind of atomic species, we found a very sharp transition to a well-faceted icosahedron with defect-free (111) surfaces, which corresponds to a low-energy shape. In contrast, in the case of particles with two atomic species, the final shape corresponds to a truncated octahedron with a very rough surface, in the sense that the surface contains many defect sites such as kinks, edges, vacancies, and di-vacancies, in agreement with the experimental observations. Our results clearly show that the effect of alloying in the case of $\mathrm{Au}-\mathrm{Pd}$ is the formation of new structures and the increase of surface roughness. Unlike the particle containing only one atomic species, in the alloy particle the resulting shape is not the most energetically lower one, but its final shape is dictated by the way the particle is cooled down. This might be a consequence of the different atomic ratio and the difference in mobility of the two atomic species from the core to the shell.

Another relevant result can be seen in the structures of Figure 6. As can be seen, the crystal in the AuPd particles is divided in "bands" of atoms with a FCC environment alternating with bands of HCP atoms. This phenomenon was first predicted by Bartell and Chushak, ${ }^{17}$ and it is expected to have a profound influence on the electric properties of the nanoparticle. This is a fundamental characteristic of the nanoparticles that may be present at all values of the number of atoms $N$ and relative concentration of the atomic species. Other authors have found a similar behavior. ${ }^{18,19} \mathrm{We}$ will discuss these findings in a more extended way elsewhere.

We conclude that producing nanoparticles with the two metals $\mathrm{Au}$ and $\mathrm{Pd}$, which are perfectly miscible, results on nanoparticles with a rough surface structure containing kinks, edges, and vacancies. We were able to identify three kinds of sites which contain a $\mathrm{Pd}$ atom surrounded by gold atoms. At these sites, the atomic positions around those atoms are distorted. These sites are the most frequently observed on the particle, and it is very likely that such sites are linked to the increase on the catalytic activity observed on these particles, since the two metals are in direct contact and this must produce a significant change in their electronic properties.

Acknowledgment. This work was supported by the International Center for Nanotechnology and Advanced Materials of The University of Texas at Austin (ICNAM), the Council for Science and Technology of the State of Nuevo León, México, and the National Council for Science and Technology, México (CONACYT), grant 43772. Computing time on the IBM Power4 resource at the Texas Advanced Computing Center at The University of Texas at Austin, is also acknowledged. Electron microscopy at the High Temperature Materials Laboratory was sponsored by the Assistant Secretary for Energy Efficiency and Renewable Energy, Office of FreedomCAR and Vehicle Technologies, Oak Ridge National Laboratory, managed by UT-Battelle, LLC, for the U.S. Department of Energy under contract number DE-AC05-00OR22725.

\section{References and Notes}

(1) Hutchings, G. J. Catal. 1985, 96, 292 4,405

(2) Haruta, M.; Kobayashi, T.; Sano, H.; Yamada, N. Chem. Lett. 1987,

(3) Enache, D.; Edwards, J.; Landon, P.; Solsona-Espriu, B.; Carley, A.; Herzing, A.; Watanabe, M.; Kiely, C.; Knight, D.; Hutchings, G. Science 2006, 311, 362. 291.

(4) Chen, M.; Kumar, D.; Yi, C.-W.; Goodman, D. Science 2005, 310,

(5) García-Gutiérrez, D.; Gutiérrez-Wing, C.; Giovanetti, L.; RamalloLópez, J.; Requejo, F.; José-Yacamán, M. J. Phys. Chem. B 2005, 109, 3813.

(6) Nosé, S. J. Chem. Phys. 1984, 81, 511.

(7) Allen, M.; Tildesley, D. Computer Simulation of Liquids; Oxford Science Publications: New York, 1987.

(8) Smith, W.; Forester, T. R. DL_POLY package of molecular simulation subroutines, 1996

(9) Rafii-Tabar, H.; Sutton, A. P. Philos. Mag. Lett. 1991, 63, 217.

(10) Rodríguez-López, J.; Montejano-Carrizales, J.; José-Yacamán, M. Appl. Surf. Sci. 2003, 219, 56.

(11) Qi, Y.; Çă̌ın, T.; Kimura, Y.; Goddard, W. A. Phys. Rev. B 1999. $59,3527$.

(12) Rafii-Tabar, H. Phys. Rep. 2000, 325, 239.

(13) Mizukoshi, Y; Fujimoto, T.; Nagata, Y.; Oshima, R.; Maeda, Y. J. Phys. Chem. B 2000, 104, 6028.

(14) Mejía-Rosales, S. J.; Fernández-Navarro, C.; Pérez-Tijerina, E.; Montejano-Carrizales, J.; José-Yacamán, M. J. Phys. Chem. B 2006, 110 12884 .

(15) Nam, H.-S.; Hwang, N. M.; Yu, B. D.; Yoon, J.-K. Phys. Rev. Lett. 2002, 89, 275502.

(16) Chushak, Y.; Bartell, L. S. J. Phys. Chem. A 2000, 104, 9328.

(17) Chushak, Y. G.; Bartell, L. S. J. Phys.Chem. B 2001, 105, 11605.

(18) Baletto, F.; Ferrando, R. Rev. Mod. Phys. 2005, 77, 371.

(19) Liu, H.; Pal, U.; Pérez, R.; Ascencio, J. J. Phys. Chem. B 2006, $110,5191$. 